\title{
Dissociated behavior of low-frequency responses and high-frequency oscillations after systemic morphine administration in conscious rats Guo-Liang Li ${ }^{a}$, Zhi-Mei Qiao, ${ }^{b, c}$, Ji-Sheng $\operatorname{Han}^{c}$ and Fei Luo ${ }^{c, d}$
}

\begin{abstract}
It has been proposed that high-frequency oscillations (HFOs) and underlying conventional somatosensoryevoked potentials (SEPs) have different brain origins. To further explore the neural mechanism of HFOs, we recorded the SEPs responding to high-intensity electrical stimulation applied to the hind paw of conscious, freely moving rats. We also investigated the effect of systemic morphine on HFOs and the conventional SEPs. HFOs after high-intensity electrical stimulation showed a widespread distribution in frontal and temporal regions of the brain. The amplitude of HFOs was significantly decreased by systemic morphine, whereas the primary conventional SEP components remained unaffected. The different changes in HFOs and primary SEP components after systemic morphine administration provided further evidence for the hypothesis that HFOs and underlying
\end{abstract}

\section{Introduction}

High-frequency oscillatory activities $(>300 \mathrm{~Hz})$ recorded in both humans and animals are drawing increasing attention on account of their unique features and possible functional roles. However, neural mechanisms underlying high-frequency oscillations (HFOs) remain unclear. Studies on somatosensory-evoked potentials (SEPs) and magnetoencephalography have revealed that HFOs are superimposed on the primary cortical response and can be extracted by digitally filtering the wide-band SEPs $(10-2000 \mathrm{~Hz})$ using a bandpass of $300-900 \mathrm{~Hz}$ [1-4]. Previous studies showed that the primary cortical response, N20, was most likely generated by excitatory postsynaptic potentials of pyramidal cells in area $3 b$ [5]. On account of the specific relationship between the primary cortical responses and HFOs, it had been proposed that they have the same source. Subsequent studies, however, suggested that HFO was an independent SEP component dissociated functionally from the primary cortical response, because it either disappeared or became significantly smaller in amplitude during sleep, whereas the N20 remained almost constant [68]. Evidences from studies on the behavior of N20 and high-frequency wavelets under varying stimulus rates [9], different stimulus intensities [10], as well as lorazepam treatment [11] also support the hypothesis that they have different origins.

Pharmacological manipulation is a useful approach to study the possible neural mechanism of biological signals. conventional SEP components have different origins. NeuroReport 21:2-7 (c) 2010 Wolters Kluwer Health | Lippincott Williams \& Wilkins.

NeuroReport 2010, 21:2-7

Keywords: conscious rat, electrical stimulation, high-frequency oscillations, morphine, somatosensory-evoked potentials

${ }^{a}$ Department of Anesthesiology, Fourth Affiliated Hospital of Hebei Medical University, Shijiazhuang, ${ }^{b}$ Department of Biomedical Engineering, School of Medicine, Tsinghua University, 'Neuroscience Research Institute and Department of Neurobiology, Peking University and ' Key Laboratory of Mental Health, Institute of Psychology, Chinese Academy of Sciences, Beijing, China

Correspondence to Dr Fei Luo, Key Laboratory of Mental Health, Institute of Psychology, Chinese Academy of Sciences, 10A Datun Road, Chaoyang District, Beijing 100101, China

Tel: +86 10 64844991; fax: +8610 64844991; e-mail: luof@psych.ac.cn

Guo-Liang Li and Zhi-Mei Qiao contributed equally to this study

Received 7 March 2009 accepted 7 May 2009

Morphine is a widely used analgesic that exerts its principal pharmacological effects on the central nervous system and produces significant effects of analgesia and sedation. Previous studies have shown that systemic morphine $(5 \mathrm{mg} / \mathrm{kg})$ preferentially attenuates the second pain mediated by unmyelinated C-fibers, whereas the first pain sensation mediated by myelinated $\mathrm{A} \delta$ nociceptive afferents is relatively insensitive [12]. Therefore, morphine should have no effect on the primary SEP components, which reflect the conduction of nerve impulses to primary sensory centers. However, it is not clear how morphine affects the high-frequency SEP components. To examine the effect of morphine on HFOs and explore the underlying neural mechanisms, we recorded the SEPs after applying high-intensity electrical stimulation to the hind paw of conscious, freely moving rats before and after morphine administration.

\section{Materials and methods}

\section{Animals and surgery}

Adult male Sprague-Dawley rats weighing 300-350 g $(n=12)$ were used in this experiment. All experiments were carried out in accordance with the Institutional Animal Care and Use Committee of Peking University Health Science Center.

Rats were anesthetized with ketamine $(100 \mathrm{mg} / \mathrm{kg}$, intraperitoneal) and fixed in a Kopf stereotaxic instrument. Twelve epidural electrodes (wired stainless-steel screws, tip diameter $1 \mathrm{~mm}$, impedance 300-350 $\Omega$ ) were 
implanted symmetrically after the exposure of the skull. These recording electrodes were arranged $3 \mathrm{~mm}$ apart from anterior to posterior. Another two electrodes were positioned 2 and $4 \mathrm{~mm}$ caudal to the lambda at the midline as the reference and ground electrodes, respectively [13]. All the screws should be attached to the dura. The location of these electrodes is shown in the inset of Fig. 1. These electrodes were connected to a twodimensional array connector and fixed to the skull with dental cement. Animals were then injected with antibiotics (penicillin, $60000 \mathrm{U}$, intramuscular) and were housed individually in cages. The rats were allowed to recover for 2 weeks.

\section{Data acquisition}

SEPs were recorded simultaneously over the 12 channels through a light-weight cable connected to a digital preamplifier. Data were recorded using an EEG/ERP system (CogniTrace ERP, ANT Inc., The Netherlands) and sampled at a rate of $2048 \mathrm{~Hz}$ together with the stimulus markers.

\section{Experimental procedures}

SEPs were evoked by applying brief electrical stimuli ( $2 \mathrm{~ms}$ duration, $6 \mathrm{~mA})$, delivered by a DS7A constant current stimulator (Digitimer Ltd, UK) to the volar surface of the left hind paw. To avoid habituation effects, low-intensity $(0.5 \mathrm{~mA})$ electrical stimuli were inserted randomly. Sixty stimuli (30 high-intensity and 30 lowintensity stimuli) were applied in a session. The interval between two sessions was fixed to $10 \mathrm{~min}$. After injecting $5 \mathrm{mg} / \mathrm{kg}$ of morphine or saline (intraperitoneal), another two sessions were delivered. The interstimulus interval was $3500-5500 \mathrm{~ms}$.

\section{Measurement of pain threshold}

Tail flick latency (TFL) in response to a radiant thermal stimulus was assessed with a $12.5 \mathrm{~W}$ projector bulb, according to the method of D'Amour and Smith [14]. The temperature was adjusted to obtain a baseline of $4-5 \mathrm{~s}$. The cut-off time was set at $15 \mathrm{~s}$. Before recording, the baseline pain threshold was measured by three tests with an interval of 5 min. Fifteen minutes after morphine or saline injection, TFLs were measured again every 5 min.

\section{Data processing}

Data epoch of $80 \mathrm{~ms}$ duration $(10 \mathrm{~ms}$ prestimulus and $70 \mathrm{~ms}$ poststimulus) was analyzed offline using the EEGLAB software [15]. For separation and isolation of

Fig. 1
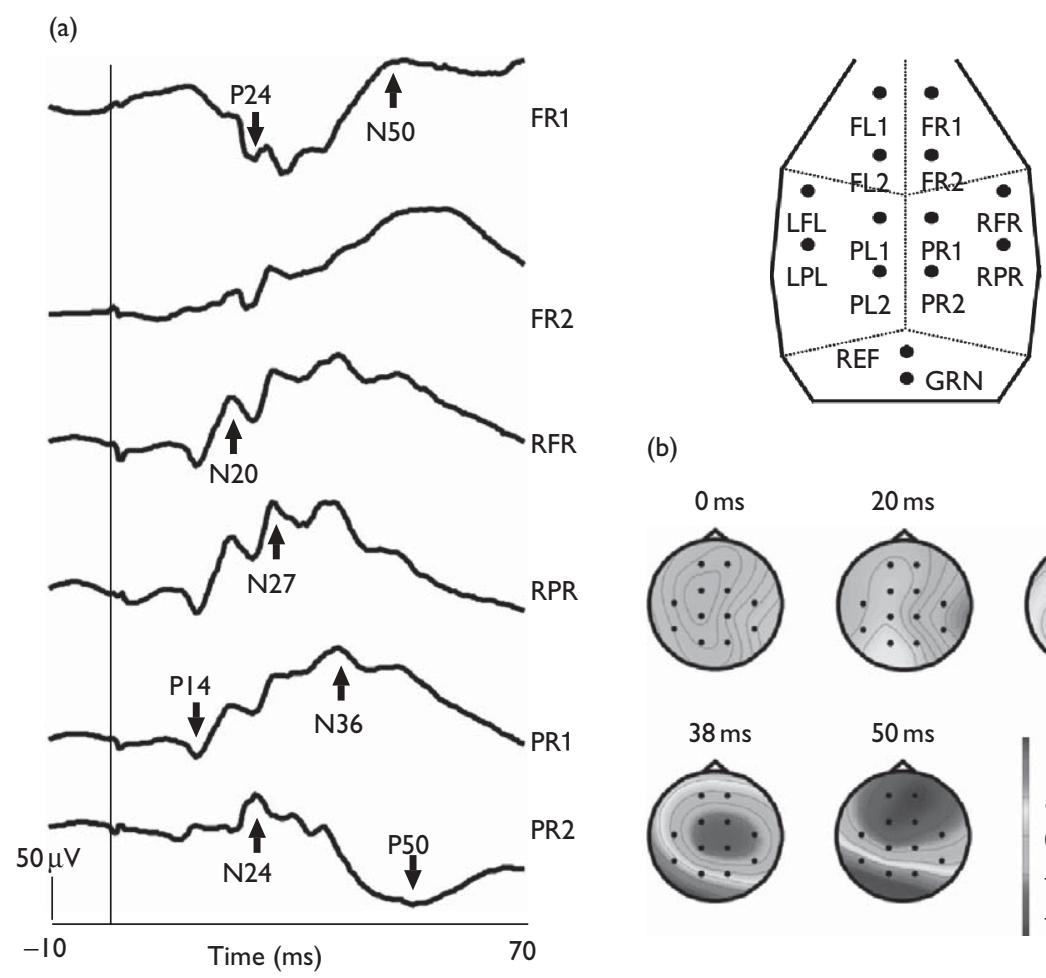

(b)
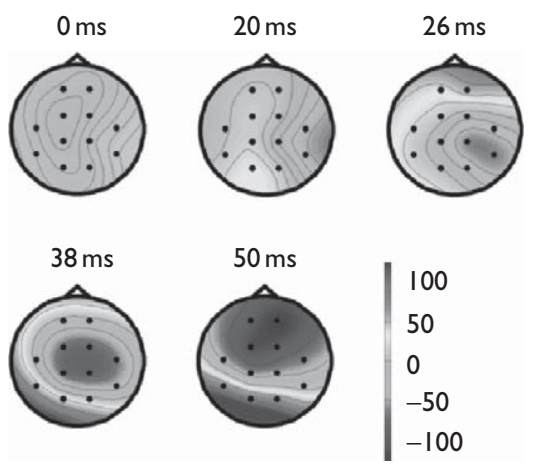

(a) Wide-band somatosensory-evoked potentials $(n=12)$ after high-intensity electrical stimulation applied to the left hind paw of a rat. Arrows pointed to the components of the primary cortical response. (b) Grand mean topographies at different timepoints poststimulus. Note that the negative current activity shifted from the contralateral temporal region $(20 \mathrm{~ms})$ to primary somatosensory cortex area $(26 \mathrm{~ms})$, then to central area ( $38 \mathrm{~ms})$ and finally located in frontal region $(50 \mathrm{~ms})$. Inset shows distribution of the 12 electrodes over the skull. FL, frontal left; FR, frontal right; LFL, left to frontal left; $\mathrm{LPL}$, left to parietal left; PL, parietal left; PR, parietal right; RFR, right to frontal right; RPR, right to parietal right; GRN, ground. 
the HFOs from original SEPs, the wide-band $(10-2000 \mathrm{~Hz})$ recorded responses were digitally filtered through a bandpass of $300-800 \mathrm{~Hz}$. Independent component analysis was used to remove possible artifact. The character of HFOs and original SEPs were evaluated on the basis of responses recorded at channel RFR. The responses that exceeded the background noise level by 3 standard deviations were considered as signals. The noise level was measured between 5 and $10 \mathrm{~ms}$ after stimulus. The number of negative peaks, the interpeak latencies, amplitudes, durations, as well as the maximal amplitudes of HFOs were measured. The interpeak latencies of HFOs were averaged with values measured from two successive negative peaks. The amplitudes of HFOs were averaged with values measured from the vertical distance from a preceding positive peak to the following negative peak. The maximal amplitudes of HFOs were averaged from the real values of the negative peaks with the maximal amplitudes (the vertical distance from the baseline to the maximal negative peaks). The duration of HFOs were measured from the onset to the offset of HFOs.

Differences in these parameters before and after morphine and saline injection were statistically analyzed using the Student's $t$-test. A value of $P$ less than 0.05 was considered to be significant.

\section{Results \\ Behavioral nociceptive responses}

All rats showed obviously nociceptive behaviors after high-intensity electrical stimuli, including immediate withdrawal of the stimulated hind paw followed by aversive behavior, such as licking accompanied occasionally by gentle biting. The TFL was prolonged obviously by morphine injection $(5.20 \pm 1.47$ vs. $14.80 \pm 1.00 \mathrm{~s}$, $P<0.001)$, whereas it remained unaffected after saline injection (4.68 \pm 0.98 vs. $4.46 \pm 1.16 \mathrm{~s}, P>0.05)$.

Table 1 Comparison of wide-band somatosensory-evoked potentials before and after morphine or saline treatment

\begin{tabular}{|c|c|c|c|c|c|c|c|c|}
\hline \multirow[b]{2}{*}{ Peak } & \multicolumn{4}{|c|}{ Latency (ms) } & \multicolumn{4}{|c|}{ Amplitude $(\mu \mathrm{V})$} \\
\hline & Pre-NS & Post-NS & Pre-Mor & Post-Mor & Pre-NS & Post-NS & Pre-Mor & Post-Mor \\
\hline P14 & $14.43 \pm 1.15$ & $14.27 \pm 1.36$ & $14.27 \pm 1.33$ & $14.49 \pm 1.36$ & $22.45 \pm 8.22$ & $22.65 \pm 7.9$ & $19.08 \pm 7.78$ & $22.99 \pm 10.25$ \\
\hline $\mathrm{N} 20$ & $20.71 \pm 1.52$ & $20.35 \pm 1.15$ & $20.47 \pm 1.11$ & $20.22 \pm 1.03$ & $-50.72 \pm 36.37$ & $-55.1 \pm 28.01$ & $-51.47 \pm 25.5$ & $-61.92 \pm 39.24$ \\
\hline P24 & $24.74 \pm 2.2$ & $24.45 \pm 1.05$ & $24.95 \pm 1.98$ & $24.41 \pm 2.19$ & $57.86 \pm 25.26$ & $67.11 \pm 35.54$ & $62.19 \pm 33.17$ & $45.83 \pm 15.17$ \\
\hline N24 & $24.85 \pm 0.79$ & $24.41 \pm 0.73$ & $24.74 \pm 0.65$ & $24.07 \pm 1.34$ & $-37.89 \pm 14.54$ & $-43.42 \pm 19.73$ & $-39.26 \pm 12.49$ & $-40.77 \pm 17.18$ \\
\hline N27 & $27.17 \pm 1.86$ & $26.68 \pm 1.68$ & $27.17 \pm 2.23$ & $26.28 \pm 3.73$ & $-75.68 \pm 33.84$ & $-75.94 \pm 24.34$ & $-66.40 \pm 18.85$ & $-60.99 \pm 21.55$ \\
\hline N36 & $36.09 \pm 2.92$ & $38.85 \pm 3.24$ & $35.73 \pm 2.25$ & $34.46 \pm 2.24$ & $-98.81 \pm 33.66$ & $-97.49 \pm 29.56$ & $-84.12 \pm 23.02$ & $-93.32 \pm 23.17$ \\
\hline N50 & $50.03 \pm 5.86$ & $49.76 \pm 6.01$ & $48.03 \pm 3.66$ & $46.48 \pm 3.07$ & $-138.60 \pm 64.22$ & $-165.45 \pm 73.31$ & $-136.19 \pm 47.41$ & $-150.27 \pm 55.29$ \\
\hline P50 & $51.52 \pm 3.98$ & $52.16 \pm 4.08$ & $51.62 \pm 6.05$ & $49.54 \pm 4.13$ & $82.67 \pm 28.53$ & $80.75 \pm 24.82$ & $78.22 \pm 25.83$ & $91.82 \pm 27.83$ \\
\hline
\end{tabular}

Post-Mor, after morphine treatment; Post-NS, after saline treatment; Pre-Mor, before morphine treatment; Pre-NS, before saline treatment.

Fig. 2

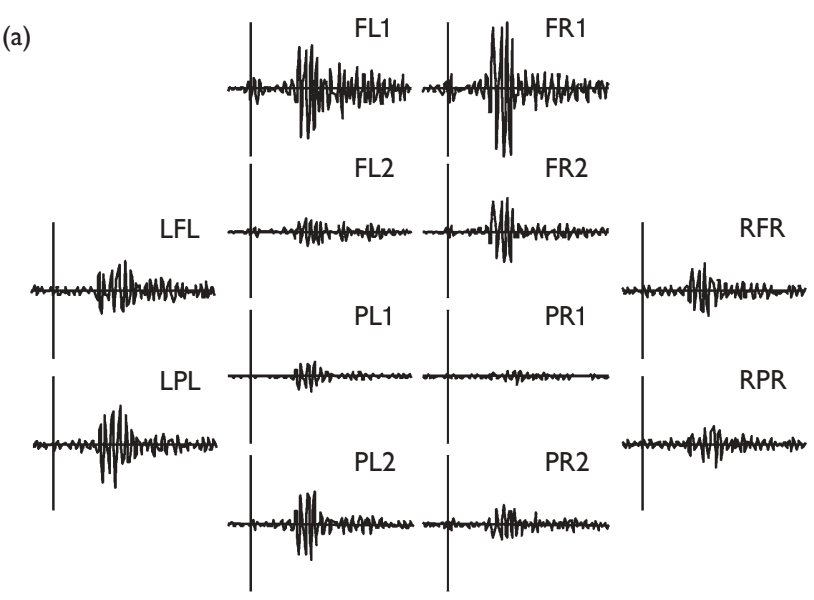

(b)

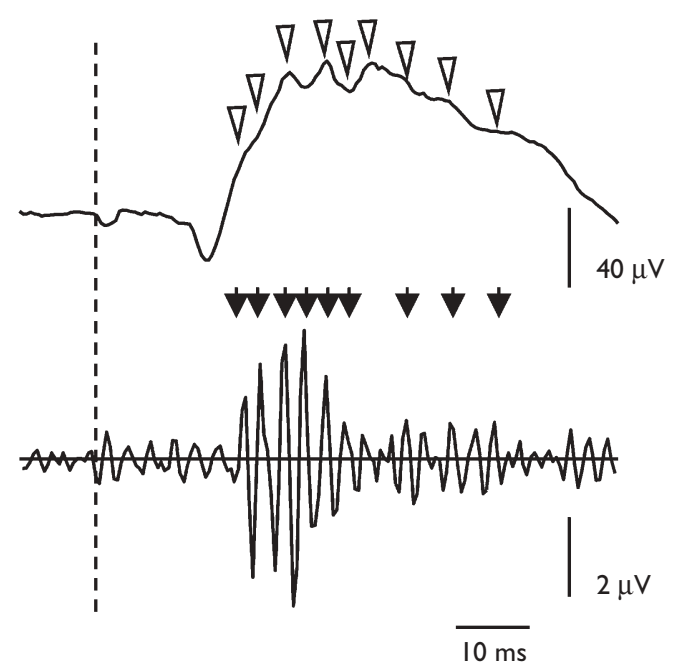

(a) An example of the high-pass-filtered $(300-800 \mathrm{~Hz})$ somatosensory-evoked potentials (SEPs) in 12 channels. High-frequency oscillation signals were extracted from traces in frontal and bilateral temporal regions. (b) The wide-band (10-2000 Hz) and high-pass-filtered (300-800 Hz) SEPs at channel RFR in a rat. Some little notches were observed on the ascending and descending slopes of the primary wide-band negative-evoked potential (upper trace). These little notches are corresponding to the high-frequency bursts in time domain (lower trace). The high-frequency bursts mainly superimposed on the ascending slope of the primary negative response. FL, frontal left; FR, frontal right; LFL, left to frontal left; LPL, left to parietal left; $\mathrm{PL}$, parietal left; $\mathrm{PR}$, parietal right; RFR, right to frontal right; RPR, right to parietal right. 


\section{Wide-band somatosensory-evoked potentials}

In all rats, primary SEP components P14, N20, N/P24, $\mathrm{N} 27, \mathrm{~N} 36$, and N/P50 could be clearly identified in traces contralateral to the stimulated side (Fig. 1a). The average latencies and amplitudes of each component in the four experimental conditions (before and after either saline or morphine injection) are shown in Table 1 .

Topographies of SEPs after stimulation are shown in Fig. 1b. At $26 \mathrm{~ms}$ poststimulus, a negative activity over the contralateral primary somatosensory cortex was observed, which then shifted to midline $(38 \mathrm{~ms})$ and then to the frontal region $(50 \mathrm{~ms})$, where it reached the maximal amplitude.

The latencies and amplitudes of all these SEP components before and after morphine or saline treatment are shown in Table 1 . There were no significant changes before and after either morphine or saline injection.

\section{High-frequency somatosensory-evoked potentials}

High-frequency oscillatory wavelets $(300-800 \mathrm{~Hz})$ that superimposed on the conventional SEPs evoked by highintensity stimulation were detected at channels over parietal and frontal regions bilaterally (Fig. 2a). In the ascending and descending slopes of the primary negative wave, several little notches were detected (Fig. 2b). These little notches temporarily corresponded to the high-frequency bursts. Parameters of HFOs at channel RFR are listed in Table 2. High-frequency bursts mainly superimposed on the ascending slope of the primary negative wave. The number of HFO bursts from the onset to the peak of the primary negative is larger than that from the peak to the endpoint (Table 2).

Systemic morphine showed a significant inhibitory effect on HFOs (Fig. 3, upper panel). The number of negative peaks, average amplitude, amplitude of the maximal negative peak, and HFO duration decreased significantly after morphine administration, whereas they remained unchanged by saline injection (Fig. 3, lower panel; Table 2).

\section{Discussion}

Cumulating evidence suggested that HFOs and conventional SEPs have different origins in the brain $[6-8,11,16]$.
In human studies, the N20 response is known to be generated in Brodmann area $3 \mathrm{~b}$ [5]. However, the origin of HFOs is still in dispute. Thalamus [17] and subthalamic sites [16] have been reported as possible sources of HFOs. Gobbelé et al. [9] suggested that HFOs reflected, at least in part, a burst of repetitive activity

Fig. 3

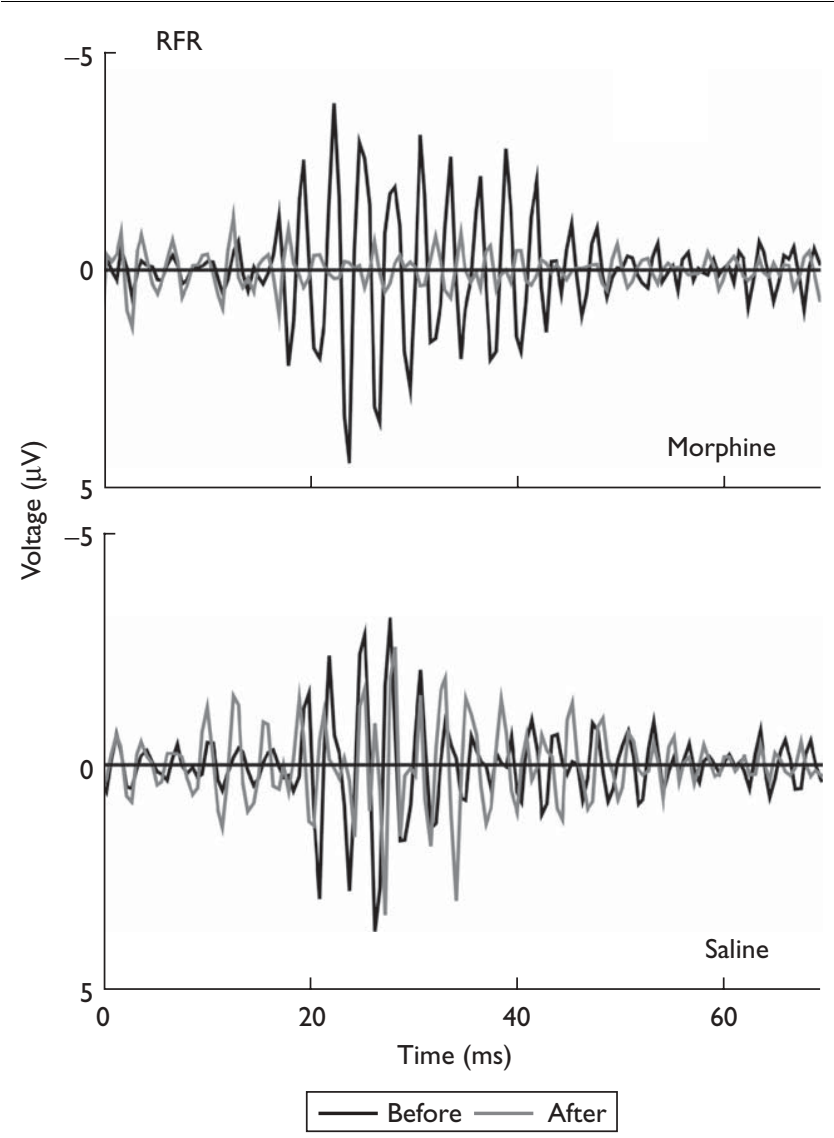

Comparison of high-frequency oscillations (HFOs) before and after morphine or saline treatment. The amplitude of HFOs after morphine injection (gray line, upper panel) was significantly smaller than that before morphine injection (black line, upper panel), while the amplitude of HFOs after saline (gray line, lower panel) did not differ significantly from that before saline injection (black line, lower panel).

Table 2 Comparison of HFOs before and after morphine or saline injection

\begin{tabular}{|c|c|c|c|c|}
\hline & \multicolumn{2}{|c|}{ Morphine } & \multicolumn{2}{|c|}{ Saline } \\
\hline & Before & After & Before & After \\
\hline Number of HFO negative peaks (total) & $10.58 \pm 2.68$ & $5.75 \pm 3.36 * *$ & $8.67 \pm 2.27$ & $8.25 \pm 4.43$ \\
\hline Number of HFO negative peaks (onset-peak) & $7.92 \pm 4.83$ & $4.83 \pm 3.16 *$ & $7.33 \pm 2.15$ & $6.67 \pm 3.11$ \\
\hline Number of HFO negative peaks (peak-endpoint) & $2.67 \pm 1.72$ & $0.92 \pm 1.16 * * *$ & $1.33 \pm 1.5$ & $1.58 \pm 2.11$ \\
\hline HFO interpeak latency (ms) & $2.49 \pm 0.54$ & $2.51 \pm 0.49$ & $2.41 \pm 0.5$ & $2.52 \pm 0.55$ \\
\hline HFO amplitude (total) $(\mu \mathrm{V})$ & $4.54 \pm 1.97$ & $3.19 \pm 1.43^{* * *}$ & $4.59 \pm 2.76$ & $5.44 \pm 3.09$ \\
\hline Maximum amplitude of HFO $(\mu \mathrm{V})$ & $3.53 \pm 1.38$ & $2.37 \pm 1.22 * *$ & $3.97 \pm 1.89$ & $4.75 \pm 2.33$ \\
\hline HFO duration (ms) & $29.67 \pm 6.64$ & $15.76 \pm 12.37 * *$ & $24.77 \pm 7.12$ & $24.01 \pm 11.53$ \\
\hline
\end{tabular}

HFOs, high-frequency oscillations.

$* P<0.05$.

$* * P<0.01$

*** $P<0.001$ vs. before 
conducted in the terminal segments of thalamocortical projection fibers initiated by the thalamic burst generator. A magnetoencephalographic study in pig detected highly synchronized repetitive spikes in thalamocortical axonal terminals and in postsynaptic intracortical cell populations [18]. In this study, electrical stimulation-evoked cerebral response distributed in the contralateral parietal area was observed at $26 \mathrm{~ms}$ poststimulus, which is similar to that found in human studies. When filtering the raw data with a high-frequency bandpass, a set of HFO wavelets was detected. We also found that systemic morphine has no effect on conventional SEP components but significantly decreased the number and amplitude of HFOs. It is well known that high-intensity electrical stimulation can activate A $\delta$ nociceptor as well as $A \beta$ mechanoreceptor. Cortical field potentials evoked by the activation of $A \delta$ nociceptor and $\mathrm{A} \beta$ mechanoreceptor cannot be affected by systemic morphine [19]. Our finding that systemic morphine has no effect on the primary SEP components agrees with previous finding. It has been proposed that supraspinal effects of morphine are primarily mediated at the thalamic level. A previous study showed that systemic morphine selectively depressed the nociceptive activity of thalamic neurons evoked either by thermal or by supramaximal percutaneous electrical stimuli [20]. The effect of morphine on HFOs observed in this study may result from the inhibitory effect of morphine on thalamus. Our results that morphine significantly decreased the number and amplitude of HFOs although having no effect on the amplitude and latency of primary SEP components provide additional evidence for their different origins.

In contrast to previous studies, we found that highintensity electrical stimulation evoked high-frequency bursts widely distributed at the bilateral frontal and parietal lobes. The widespread distribution of HFOs found in this study may be because of the fact that the stimulus intensity applied in previous studies was greatly smaller than that used in this study.

The different behaviors of HFOs and conventional SEPs after morphine treatment indicate that they have different physiological functions. Halboni et al. [8] proposed that the high-frequency thalamic or cortical activity reflects a somatosensory arousal system. In this study, the randomly inserted low-intensity stimulation evoked only low-amplitude HFOs in the parietal channels (data not shown), whereas widely distributed HFOs with high amplitude were evoked after high-intensity electrical stimulation. The amplitude of HFOs evoked by high-intensity stimulation was reduced dramatically by systemic morphine. The higher amplitude and wide distribution of HFOs after high-intensity stimulation and the dramatic reduction of HFOs after morphine both suggested the vigilance-dependent property of HFO.
We speculate that conventional early SEPs reflect the location of stimulus, whereas HFOs reflect the cortical arousal elicited by stimulation.

\section{Conclusion}

In conclusion, dissociated behaviors of HFOs and conventional SEPs after systemic morphine administration were observed in this study, indicating different origins. These findings implicate that HFOs and conventional SEPs are functionally different in sensory processing.

\section{Acknowledgement}

This work was supported by National Natural Science Foundation of China (30570577 and 30770688) to F.L.

\section{References}

1 Curio G, Mackert BM, Burghoff M, Koetitz R, Abraham-Fuchs K, Harer W. Localization of evoked neuromagnetic $600 \mathrm{~Hz}$ activity in the cerebral somatosensory system. Electroenceph Clin Neurophysiol 1994; 91:483-487.

2 Hashimoto I, Mashiko T, Imada T. Somatic evoked high-frequency magnetic oscillations reflect activity of inhibitory interneurons in the human somatosensory cortex. Electroenceph Clin Neurophysiol 1996; 100:189-203.

3 Ozaki I, Suzuki C, Yaegashi Y, Baba M, Matsunaga M, Hashimoto I. High frequency oscillations in early cortical somatosensory evoked potentials. Electroenceph Clin Neurophysiol 1998; 108:536-542.

4 Ozaki I, Hashimoto I. High-frequency oscillations and underlying early cortical responses in SEPs and SEFs. Int Congr Ser 2002; 1232:175-180.

5 Allison T, McCarthy G, Wood CC, Darcey TM, Spencer DD, Williamson PD. Human cortical potentials evoked by stimulation of the median nerve. I. Cytoarchitectonic areas generating short-latency activity. J Neurophysiol 1989; 62:694-710.

6 Emerson RG, Sgro JA, Pedley TA, Hauser A. State-dependent changes in the N20 component of the median nerve somatosensory evoked potential. Neurology 1988; 38:64-67.

7 Gobbelé R, Waberski TD, Kuelkens S, Sturm W, Curio G, Buchner H. Thalamic and cortical high-frequency $(600 \mathrm{~Hz})$ somatosensory-evoked potential (SEP) components are modulated by slight arousal changes in awake subjects. Exp Brain Res 2000; 133:506-513.

8 Halboni P, Kaminski R, Gobbelé R, Züchner S, Waberski TD, Töpper R, et al. Sleep-stage dependent changes of high-frequency part of the somatosensory evoked potentials at the thalamus and cortex. Clin Neurophysio/ 2000; 111:2277-2284.

9 Gobbelé R, Buchner H, Curio G. High-frequency $(600 \mathrm{~Hz})$ SEP activities originating in the subcortical and cortical human somatosensory system. Electroenceph Clin Neurophysiol 1998; 108:182-189.

10 Klostermann F, Nolte G, Losch F, Curio G. Differential recruitment of high frequency waveletes $(600 \mathrm{~Hz})$ and primary cortical response (N20) in human median nerve somatosensory evoked potentials. Neurosci Lett 1998; 256:101-104.

11 Restuccia D, Valeriani M, Grassi E, Mazza S, Tonali P. Dissociated changes of somatosensory evoked low-frequency scalp responses and $600 \mathrm{~Hz}$ bursts after single-dose administration of lorazepam. Brain Res 2002; 946:1-11.

12 Cooper BY, Vierck CJ, Yeomans DC. Selective reduction of second pain sensations by systemic morphine in humans. Pain 1986; 24:93-116.

13 Shaw FZ, Chen RF, Tsao HW, Yen CT. A multichannel system for recording and analysis of cortical field potentials in freely moving rats. $J$ Neurosci Meth 1999; 88:33-43.

14 D'Amour FE, Smith DL. A method for determining loss of pain sensation. J Pharmacol Exp Ther 1941; 72:74-79.

15 Delorme A, Makeig S. EEGLAB: an open source toolbox for analysis of single-trial EEG dynamics including independent component analysis. J Neurosci Meth 2004; 134:9-21. 
16 Gobbelé R, Waberski TD, Simon H, Peters E, Klostermann F, Curio G, et al. Different origins of low- and high-frequency components $(600 \mathrm{~Hz})$ of human somatosensory evoked potentials. Clin Neurophysiol 2004; 115:927-937.

17 Eisen A, Roberts K, Low M, Hoirch M, Lawrence P. Questions regarding the sequential neural generator theory of the somatosensory evoked potential raised by digital filtering. Electroenceph Clin Neurophysiol 1984; 59:388-395.
18 Ikeda H, Leyba L, Bartolo A, Wang Y, Okada Y. Synchronized spikes of thalamocortical axonal terminals and cortical neurons are detectable outside the pig brain with MEG. J Neurophysio/ 2002; 87:626-630.

19 Abdulla F, Aneja IS, Bhargava KP. Effect of morphine on far-field somatosensory evoked potentials in the rat. Neuropharmacology 1989; 28:69-73.

20 Monconduit L, Bourgeais L, Bernard JF, Villanueva L. Systemic morphine selectively depresses a thalamic link of widespread nociceptive inputs in the rats. Eur J Pain 2002; 6:81-87. 